\title{
Notas epistemológicas en torno a la politica de cifras de la violencia contra las mujeres ${ }^{1}$
}

\author{
Epistemological notes on the politics of numbers on violence \\ against women
}

\author{
Cecilia Varela \\ Investigadora adjunta del CONICET \\ Universidad de Buenos Aires. \\ Catalina Trebisacce \\ Universidad de Buenos Aires. \\ catalinaptrebisacce@gmail.com (ARGENTINA)
}

Recibido: 28.12 .2019

Aceptado: 19.11.2020

\section{RESUMEN}

En el presente trabajo nos proponemos abordar la política de cifras desplegadas por los movimientos de mujeres en Argentina de la última década en torno a las violencias contra las mujeres. En un contexto de expansión de los feminismos en el país y una creciente institucionalización de perspectivas que se reconocen en esa matriz, la retórica de las cifras se ha convertido en la lingua franca para la visibilización de las situaciones de violencia contra las mujeres. Las preguntas que nos formulamos son las siguientes: ¿Cómo se organiza hoy el saber sobre la violencia de género? ¿Cómo operan las cifras en la construcción de ese saber y en su difusión e impacto social? Tomando como corpus para la indagación el registro de femicidios de la Casa del Encuentro nos interesa, por un lado, detenernos en los supuestos epistemológicos y las elecciones metodológicas de su confección y, por el otro, abordar los procesos sociales de construcción y validación de cifras en torno a los femicidios en un contexto de despliegue de políticas de cifras. Nuestro argumento es, por un lado, que el registro despliega un método positivista inductivo que actúa ficciones de objetividad científica a

\footnotetext{
1 Este artículo es el resultado de indagaciones realizadas desde el año 2018. Parte de este trabajo fue publicado en una versión previa en Los feminismo en la encrucijada del punitivismo, Biblios, 2020.
} 
partir del empleo de las cifras como evidencia indiscutible de los "hechos". Por el otro lado, sostenemos que un saber en torno a los femicidios se legitima como experto a partir de la política de cifras.

\title{
PALABRAS CLAVE
}

Política de cifras, violencia de género, feminismos

\begin{abstract}
In this paper we aim to address the politics of numbers displayed by women's movements in Argentina in the last decade about violence against women. In a context of expansion of feminisms across the country and a growing institutionalization of the gender perspective, the rhetoric of numbers has become the lingua franca for the visibility of situations of violence against women. The questions we ask ourselves are: How is the knowledge about gender violence organized today? How do the figures operate in the construction of this knowledge and in its dissemination and social impact? Taking as a corpus the register of femicides of Casa del Encuentro, we are interested, on the one hand, in interrogate the epistemological assumptions and the methodological choices behind it and, on the other, to address the social processes of construction and validation of figures around femicides in a context of politics of numbers. Our argument is, on the one hand, that the register displays an inductive positivist method that acts fictions of scientific objectivity based on the use of figures as indisputable evidence of «facts». On the other hand, we argue that knowledge about femicides is legitimized as expert based on the politics of numbers.
\end{abstract}

\section{KEY WORDS}

Gender violence, feminisms, politics of numbers

¿Con la sangre de quien se crearon mis ojos? Donna Haraway, 1995: 330

\section{INTRODUCCIÓN}

En las últimas décadas investigaciones antropológicas se han visto comprometidas en volver reconocibles los distintos modos por los que las sociedades occidentales contemporáneas consiguen identificar y significar diferentes experiencias sociales, interpersonales e íntimas, como vivencias violentas demandando así atención e impugnación social. Estas investigaciones, que van 
conformando un incipiente campo de estudio específico, son un territorio estratégico para pensar discursos políticos y sociales actuales, señala Rifiotis (2015 [1997]). En dichos discursos, la "violencia" prolifera como una supervivencia arcaica que debe ser domesticada pero también emerge como un signo del presente identificado con la desagregación social y la amenazante multiplicación de sus formas (Garriga Zucal y Noel, 2010; Gatti, 2017). Ella concita sentimientos de indignación y condena, y es el parámetro a partir del cual discutimos la legitimidad de prácticas y relaciones en el tiempo presente. La "violencia" aparece como el marcador del límite civilizatorio, su exterior constitutivo (Elías, 2009). Como sostiene Tonkonoff (2004), este significante estructura las prohibiciones fundamentales de un orden socio-simbólico determinado. Cuando se alza la voz de alerta de la violencia, se denuncia que ha tenido lugar una fractura de la frontera civilizatoria. En el último tercio del siglo veinte esa denuncia ha cambiado parcialmente las condiciones de existencia de diversas poblaciones: animales, niñxs, mujeres, entre ellos.

Distintos trabajos han abordado cómo en distintas latitudes y contextos, desde los años ochenta, el movimiento feminista demandó que distintas prácticas -antaño incuestionadas en su legitimidad- fueran ahora concebidas e impugnadas como violencia hacia las mujeres, (luego violencia de género) (Álvarez, 2003; De Miguel Álvarez, 2005; Gregori, 2010; Daich, 2012). Hacia los años 90s la campaña anti violencia logró traducir distintas experiencias de opresión - en muy distintos contextos - en el lenguaje transnacionalizado de los derechos humanos a través de sus intervenciones en foros supranacionales y transnacionales. $\mathrm{Al}$ ocaso del siglo XX, podemos hablar ya de la conformación de un régimen de verdad de la violencia de género, es decir, un conjunto de saberes, normativas, burocracias y discursos sociales, políticos, científicos y massmediáticos orientados a determinar qué debe de ser entendido por violencia de género y qué intervenciones reclama. Es un régimen expansivo que permanentemente identifica, define y amplía las incumbencias y las modalidades en las que la violencia de género se expresa. Se trata de un dispositivo social complejo de denuncia del cual participan tanto organizaciones de la sociedad civil como instancias gubernamentales, en un interjuego de planos nacionales, transnacional y supranacionales.

En el caso argentino, la introducción de la perspectiva de género ligada a un discurso de derechos se inicia hacia fines de los años ochenta, pero experimenta una aceleración en los últimos quince años al compás del crecimiento del movimiento feminista local y la consolidación de sus articulaciones transnacionales. En este proceso de institucionalización la agenda de la violencia de género fue adquiriendo progresivamente un lugar central, mientras que otros temas -como los derechos no reproductivos- fueron relativamente relegados.

En este trabajo nos interesa detenernos en ciertas formas de producción de saber que resultan privilegiadas dentro del régimen de la violencia de género por su capacidad de dar cuenta, en el doble sentido, de hacer inteligible y de volver contable un problema social. Estos saberes construidos trasnacionalmente elaboran mediciones y poniendo en circulación cifras, como modo en que distintos 
actores interesados modelan una conciencia pública sobre un problema social. El concepto política de cifras de Andreas y Greenhill (2010) es el que nos permite captar esta dimensión productiva de los números.

En el contexto argentino, en los últimos diez años, la cifra de los femicidios logró instalarse como el indicador por excelencia de la violencia hacia las mujeres. Ello no necesariamente por sus virtudes metodológicas para dar cuenta de la condición de vida de las mujeres sino por la legitimidad social adquirida a partir de la difusión y diseminación de la denuncia cifrada producida desde el activismo feminista y el movimiento amplio de mujeres. En este trabajo nos proponemos abordar la política de cifras desplegada por una ONG central en la construcción de una experticia feminista en la última década. Se trata de la Casa del Encuentro, una organización creada en el 2003 y radicada en la capital del país que tiene por objetivo declarado la lucha por los derechos humanos de las mujeres. Desde el año 2008 hasta el presente es la ONG que más se ha destacado por la producción de informes sobre la violencia extrema hacia las mujeres. En diciembre de 2015, con el ascenso de un gobierno de derecha, Fabiana Tuñez, quien era la directora ejecutiva de la ONG, pasó a ser la directora del Consejo Nacional de las Mujeres de la Nación.

Las preguntas que nos formulamos son las siguientes: ¿Cómo se organiza hoy el saber sobre la violencia de género? ¿Cuáles son las categorías que estructuran ese conocimiento? ¿Qué matriz de inteligibilidad moldean? ¿Cómo operan las cifras en la construcción de ese saber y en su difusión e impacto social? Para abordar estas indagaciones nos proponemos analizar los supuestos epistemológicos y las elecciones metodológicas implicadas en la construcción de cifras en torno a femicidios, por un lado. Y, por otro, abordar los procesos sociales de construcción y validación de cifras en torno a los femicidios en un contexto de producción de políticas de cifras (Andreas-Greenhill, 2010). En el tránsito de estas indagaciones consideramos algunos estudios sobre la producción de un saber en torno a la violencia de género realizados por Osborne $(2008,2009)$, Casado Aparicio (2012) y García Selgas y García García (2008) para el contexto español.

En las siguientes páginas sostendremos que un saber en torno a los femicidios se legitima como experto a partir de la política de cifras. La producción de este saber experto está enmarcada en un proceso más general de progresiva institucionalización del feminismo, tanto en el norte como en el sur global, bajo la denominada perspectiva de género que algunas autoras han analizado como la emergencia de una gobernanza feminista (Halley-Kotiswaran-Thomas-Shamir, 2006). El período que abordamos corresponde a una etapa vibrante, de multiplicación de voces de la sociedad civil que encontraron una plataforma de amplificación en los medios de comunicación. Asimismo, argumentaremos que dicho saber se produce a partir de una serie de prácticas que ensayan una metodología que permiten actuar ficciones de objetividad científica.

El corpus empírico sobre el que trabajaremos está conformado por el informe de femicidios producido por la Casa del Encuentro en el año 2013 que compila y analiza la información por ellas mismas producida desde el año 2008. 
Nuestra indagación se construye a partir del entrecruzamiento de un conjunto de reflexiones en torno al poder de las cífras en las sociedades contemporáneas, los aportes producidos por los estudios de violencia arriba comentados y una perspectiva epistemológica feminista.

\section{LAS CIFRAS, UN TALISMÁN MODERNO}

Al compás de la consolidación de los estados nación modernos y el proyecto de expansión colonial, los datos estadísticos se constituyeron en una herramienta para el gobierno de poblaciones. La estadística fue el medio empleado y el saber privilegiado para dar cuenta de las regularidades de las poblaciones. Un conocimiento técnico que demostraba que fenómenos que podrían suponerse irregulares, azarosos, fruto de decisiones individuales o coyunturales respondían en realidad a ciclos esperables de la naturaleza de las poblaciones humanas pasibles de cuantificación (Foucault, [1978] 2006). A mediados del siglo XX estas mediciones se vieron potenciadas por la posibilidad técnica de procesar grandes volúmenes de datos (García Ferrando, 1996). Y más recientemente, hacia la década del 90 los organismos internacionales se interesaron por la producción de indicadores de alcance global, que demandaron el relevamiento de información en nuevas áreas en distintos contextos locales a cargo de una experticia (Engle Merry, 2016)

Pero la producción de cifras no ha sido una actividad privativa de instancias de gobierno nacional o supranacional, sino que tempranamente las cifras fueron reconocidas como una estrategia para la construcción y legitimación de problemas en la agenda pública por parte de distintos actores de la sociedad civil. Andreas y Green Hill (2010) han propuesto el concepto de política de cifras para captar el conjunto de acciones que crean, seleccionan, promulgan y diseminan números por parte de actores interesados en definir un problema público.

Para Poovey (1998) las cifras resultan paradigmáticas de la construcción del "hecho moderno" del siglo XIX que aparenta escindir interpretación de descripción, privilegia cantidad sobre cualidad y equivalencia sobre diferencia. Las cifras son aún persuasivas y, en cierto sentido, es comúnmente reconocida la capacidad de manipulación que ellas poseen. Pero, simultáneamente y paradójicamente, son consideradas el instrumento que brinda un conocimiento libre de valores. Engle Merry (2016) afirma que la cuantificación provee un conocimiento simple para fenómenos complejos bajo un aura de neutralidad que borra el trabajo interpretativo de su construcción. Sin embargo, para esta autora la producción de cifras no se agota en una cuestión de conocimiento sino que es una tecnología articulada a emergentes formas de gobierno en la contemporaneidad.

Las cifras detentan poderes "mágicos" y encarnan representaciones poderosas de la verdad (Best, 2001) Así quienes emprenden la tarea de modelar la conciencia pública en torno a algún problema deben de rendirles algún culto para validarse como interlocutores. Por otro lado, los recaudos epistemológicos 
planteados eventualmente por quienes las construyen se desvanecen toda vez que comienzan a rodar en el debate público.

En nuestro caso las cifras evidencian ser un asunto altamente político, en torno al cual orbitaron distintos actores que disputaron una posición experta en su producción. En el tiempo que va desde el lanzamiento de los primeros informes de la Casa del encuentro hasta las desbordantes convocatorias del NUM, la necesidad de disponer de cifras para hablar de las violencias hacia las mujeres se convirtió en un consenso extendido dentro del movimiento amplio de mujeres. Al institucionalizarse la demanda, ésta se traduce en distintas iniciativas de registro por parte de distintas burocracias estatales. A partir de aquí el Estado pareciera aceptar una conceptualización de la problemática en términos de cifras. Nos preguntamos por los límites de esta estrategia y por el cuento que cuentan los números, las interpretaciones que ellos arrastran.

\section{LA VIOLENCIA DE GÉNERO: DEL RETORNO A LA DEMOCRACIA AL NI UNA MENOS}

A partir del retorno a la democracia, y en sintonía con instancias supranacionales, se instala y configura, con progresiva legitimidad en la agenda pública, la noción de la existencia de una problemática específica de las mujeres que reclama atención y tratamiento por parte del Estado. Para el caso argentino, como en otros contextos, la conformación de un problema público específico en torno a la violencia contra las mujeres supuso una activa (y progresiva) diferenciación del paradigma de la violencia doméstica ${ }^{2}$, así como también un trabajo de reorientar las interpretaciones que observaban acontecimientos fortuitos o pasionales para conseguir señalar las relaciones estructurales de desigualdad que los sustentaban, construidas sobre diferencias de género. La creación de la Subsecretaría de la Mujer en 1987 (luego Consejo Nacional de las Mujeres), la llamada ley de cupos de 1991 y la modificación de la Constitución Nacional de 1994 que otorgó jerarquía constitucional a las convenciones internacionales en esta temática, dan cuenta de este proceso. En el año 2006 se lanzó el programa "Las víctimas contra la violencia" que si bien no se circunscribía a mujeres, pues también atendía casos de niñxs, proponía una intervención desde un paradigma actualizado en temáticas de género. En el 2009, la ley 26.485 de "Protección Integral para prevenir, sancionar y erradicar la violencia contra las mujeres en los ámbitos en que desarrollen sus relaciones interpersonales" reconoce la misma perspectiva de tratamiento para la problemática. ${ }^{3}$

\footnotetext{
${ }^{2}$ Aunque la Ley 24.417 de Protección contra la violencia familiar sancionada en 1996 introducía efectivas medidas de protección para las mujeres conservaba la perspectiva de la violencia con asunto familiar.

${ }^{3}$ Como antecedente de este nuevo enfoque en las políticas públicas podemos mencionar la pionera Ley Orgánica 1/2004, de medidas de protección integral contra la violencia de género, aprobada por el estado español.
} 
La preocupación en torno a la violencia hacia las mujeres fue un asunto destacado en la militancia feminista de la ciudad de Buenos Aires desde comienzos de los ochenta (Trebisacce, 2020), pero fue en el nuevo milenio donde este asunto cobró especial interés en distintas agrupaciones de la sociedad civil entre las que consiguió un rol protagónico la Casa del Encuentro. Esta organización, desde sus inicios, se constituyó como un espacio de reflexión y de encuentro para la comunidad lésbica con una perspectiva feminista. A partir del año 2007 se produce un viraje en sus líneas de trabajo y comienza a denunciar casos de trata de mujeres. Finalmente, en el 2008 comenzó a contabilizar los casos de femicidios y en 2009 -tras recibir financiamiento de la Embajada Norteamericana y la Fundación Avon- tomó la tarea de producir un informe anual a partir de los casos que hubieran aparecido en la prensa. Esta estrategia ya había sido desplegada por las organizaciones feministas en otras partes del mundo, tal como lo hizo la Federación de Mujeres Separadas y Divorciadas en España para discutir las estadísticas oficiales de violencia contra las mujeres desde fines de la década del noventa (Osborne, 2008).

La Casa del Encuentro, compuesta por militantes y profesionales del derecho y la psicología, eminentemente, se convirtió así en la primera asociación civil argentina en producir este dato que rápidamente fue difundido por medios alternativos, oficiales y hegemónicos. Esta ONG pasó a ser el referente estadístico más importante hasta la actualidad, aún a pesar de las limitaciones metodológicas abiertamente reconocidas (tales como contar únicamente con las fuentes periodísticas) y de que el Estado, desde el 2015, produce sus propias estadísticas.

La instalación del paradigma de la violencia contra las mujeres también fue tomando forma en los medios masivos de comunicación, en lo que puede leerse como una convergencia de intereses entre periodistas, feministas y las empresas de los medios de comunicación. Así se hicieron cada vez más frecuentes noticias sobre supuestos casos de trata de personas, primero, y asesinatos a mujeres caratulados como femicidios, luego. Entre la denuncia y la espectacularización resonaron decenas de nombres de las mujeres asesinadas. Finalmente, en el año 2012 se profundizó el camino de reconocimiento de la violencia de género en el campo penal al modificarse el artículo 80 del Código Penal que reconoce un nuevo tipo de agravante vinculado al género para los casos de homicidios de mujeres, ${ }^{4}$ más conocido como delito de femicidio $^{5}$.

Como expresión de una nueva sensibilidad (legal, social y massmediática) en torno a la violencia contra las mujeres, deben leerse las desbordantes movilizaciones del colectivo Ni una Menos (NUM). El NUM estaba conformado por un grupo de periodistas y activistas de renombre que consiguieron propagar el men-

${ }^{4}$ Ley 26.791.

${ }^{5}$ La categoría femicidio fue originalmente propuesta en 1976 por Diana Russell en el contexto del Tribunal Internacional de crímenes contra las mujeres para testificar sobre los asesinatos misóginos. Durante la década del 90 la categoría fue retomada y sujeta a reelaboraciones por parte de feministas latinoamericanas (Lagarde 2008, 2011; Carcedo-Sagot 2000; Segato 2006; Monárraez 2009). Los crímenes de ciudad Juárez fueron un laboratorio de discusión respecto de sus alcances, posibles clasificaciones y límites. 
saje de denuncia y el llamado a la movilización por los medios masivos. En la primera movilización que organizaron para el 3 de junio, se acercaron a la plaza aproximadamente 300.000 personas y más de 250000 se movilizaron en distintas provincias. Las dimensiones de las manifestaciones fueron una sorpresa para periodistas, políticos/as, y analistas, como también para las propias organizadoras.

Con miras a la convocatoria a la movilización fueron elaboradas una serie de demandas que iban desde la instrumentación de la ley de Protección integral de las mujeres hasta la capacitación de operadores judiciales. En el segundo punto de aquella serie destacaba el pedido de confección de estadísticas oficiales de femicidios en Argentina que continuaran institucionalmente el trabajo que venía produciendo la Casa del Encuentro. ${ }^{6}$ Este pedido fue el que encontró más rápidas respuestas. Dos días después representantes del Ministerio de Justicia y Derechos Humanos se reunieron con algunas de las periodistas convocantes y la directora de la Casa del Encuentro, para analizar las demandas y concluyeron en la importancia de la producción de cifras. A partir de allí a través de la Resolución 1449/2015, con fecha 5 de junio de 2015, se crea la Unidad de Registro, Sistematización y seguimientos de femicidios y homicidios agravados por el género dependiente del Ministerio de Justicia y Derechos Humanos. No fue la única dependencia estatal que se hizo eco de la manifestación. Desde la Corte Suprema, y bajo la órbita de la Oficina de la Mujer, se crea en esos mismos días el Registro de Femicidios de la Justicia Argentina. A través de la instalación de esta política de cifras distintas burocracias estatales admitían la existencia del problema y que parte de la solución del mismo estaba ligado a la producción de un saber cifrado al respecto. ${ }^{7}$

Por su parte, distintas organizaciones de la sociedad civil, evidenciando una desconfianza a las burocracias oficiales, emprendieron la tarea de producir registros propios de los casos de femicidios. A partir de ese mismo año y los sucesivos proliferaron observatorios como el Instituto de Políticas de Género Wanda Taddei (2015), el Registro Nacional de Femicidios de Mujeres de la Matria Latinoamericana (2016) vinculado a la agrupación MuMaLa y el Observatorio de Géneros y Políticas de la Ciudad (2016) en relación a la agrupación Mala Junta-Patria Grande, entre otros. La proliferación de estos observatorios organizados bajo el modelo de La Casa del Encuentro (observatorio Adriana Marisel Zambrano, 2009) evidencia la eficacia política (y la potencia talismánica) que adquirieron los números para el tratamiento de la violencia de género y la construcción del régimen de su verdad contemporáneo.

${ }^{6}$ Documento Ni Una Menos disponible online en: https://tn.com.ar/sociedad/niunamenosel-documento-completo-en-contra-del-femicidio 594509 (consultado el 24 de octubre de 2018).

${ }^{7}$ En 2017, el Instituto Nacional de Estadísticas y Censos de la República (INDEC) creó el propio Registro Único de la Violencia contra las Mujeres. 


\section{LA VOLUNTAD DE CONTAR: DE LA IGNORANCIA AL CÁLCULO PREDICTIVO}

El 25 de noviembre de 2013, en el día de lucha contra la violencia de género, La Casa del Encuentro presentó su informe "Por Ellas. 5 años de informes de femicidios" en la residencia del Embajador de los Estados Unidos, junto a las presencias de representantes de las Naciones Unidas y de la Fundación Avon quienes, en distintas instancias, habían financiado el proyecto.

En sus primeras páginas el informe que reunía la información recopilada en los últimos cinco años, presentaba sus objetivos de la siguiente manera:

Nuestro primer objetivo fue precisamente instalar este concepto [de femicidio], como un impulso, como un aporte desde la sociedad civil, para promover y generar más políticas públicas de prevención, sensibilización, asistencia y sanción, para disminuir hasta erradicar los asesinatos de mujeres, víctimas de la violencia de género. (...) En nuestra planificación se hizo evidente la necesidad de contar con Estadísticas Oficiales sobre una de las formas de violencia contra las mujeres, más graves de nuestra sociedad, incluyendo un monitoreo que dé cuenta de cada lugar de nuestro país, con cifras que revelen la dimensión real de esta gravísima problemática. Ante la completa falta de estadísticas, nos propusimos como objetivo que, hasta tanto se generen, comenzaríamos a trabajar con los casos publicados en los medios de comunicación, como única fuente de información posible para nosotras, y aunque comprendíamos, que sólo lograríamos un corte sesgado de esta realidad, igualmente sabíamos que sería un aporte para comenzar a generar conciencia y a trabajar para propiciar un cambio cultural (2013:15).

La cifra de femicidios que recogía La Casa del Encuentro a partir de las noticias publicadas en medios de prensa se proyectaba así contra la "ausencia de estadísticas oficiales", la cual era interpretada como un obstáculo para la construcción de políticas públicas capaces de proteger a las mujeres. Con la confección del informe se producía lo que Tuana (2006) denominó una epistemología de la ignorancia, es decir, la delimitación y producción de vacíos, lagunas y ausencias de conocimiento que posibilitaban (y reclamaban) la emergencia de un conocimiento que las contrarrestara. De este modo, bajo esta perspectiva, conocimiento e ignorancia están mutuamente implicados. Las "lagunas de conocimiento" son activamente producidas en el mismo proceso de construcción de saber experto que las combate. La reflexión aquí no atañe a la verdad o falsedad de los conocimientos producidos sino a su proceso de legitimación y valoración social. Lxs expertxs emergen aquí como los agentes capaces de identificar y detectar las lagunas, capaces de disolverlas por intermedio de la producción de nuevos conocimientos. En este sentido, La Casa del Encuentro fue adquiriendo los rasgos de una ONG profesionalizada cuyas integrantes supieron construir, a través de la política de cifras, un camino para modelar su propia experticia y posición en el campo. ${ }^{8}$ Tras la noción de que el vacío debía llenarse con mediciones y estadísti-

${ }^{8}$ Diversas autoras han estudiado el proceso de oenegización que atravesó el campo de las 
cas anidaba el presupuesto de que las cifras constituyen la vía regia para conocer y explicar el mundo social.

El observatorio Adriana Maricel Zambrano se constituyó para lidiar con las "lagunas de conocimiento". Militantes y voluntarias se ocuparon de realizar un seguimiento de diarios nacionales y provinciales (en un primer momento $43 \mathrm{y}$ a partir de 2010, 210 diarios), poniendo de relieve todas las noticias en las que se hiciera referencia a asesinatos de mujeres. Las noticias eran analizadas en función de los datos que la crónica policial brindaba. Y al finalizar cada año se presentaba un informe del relevamiento realizado. En el año 2013 se compiló un informe más general que recuperaba el trabajo de cinco años de observatorio y que analizamos en esta ocasión.

La Casa del Encuentro desplegó un método ya ensayado en otras geografías que, aunque precario y desprovisto de protocolos, se orientaba a practicar los imaginados pasos en la producción científica de cifras. La construcción de un Observatorio fue el dispositivo creado para tal fin. En esta práctica desarrollaron lo que llamaremos procedimientos performáticos de objetividad: observar, cuantificar y visibilizar. Estos procedimientos constituyen tres dimensiones de las actividades del observatorio, sólo deslindables analíticamente.

Decimos que se trata de procedimientos en tanto que son un modo de hacer estabilizado. Y sostenemos que son performáticos por la capacidad que detentan para constituirse en prácticas que generan y garantizan su propia legitimidad (y la de sus resultados), a efecto de dinámicas de identificación/imitación con las representaciones circulantes de los procesos de investigación científica. De modo que, podríamos decir que los procedimientos performáticos de objetividad que sostienen los observatorios actúan las fantasías del método y terminan encarnándola. Cada uno de los tres procedimientos que identificamos (observar, cuantificar y visibilizar) detenta una serie de supuestos empiristas e inductivos que aportan legitimidad científica al régimen de verdad de la violencia de género.

El procedimiento de observar se vincula directamente a aspiraciones de exhaustividad que intentan contrarrestar la fantasmagoría de un subregistro estructural. Así, lo que se despliega a lo largo de los informes de La Casa del Encuentro no es tanto una preocupación por deslindar qué es y qué no es un femicidio, o cuáles son las condiciones sociales y los ciclos vitales asociados a su ocurrencia, sino que importa capturar "más peces en la red", contabilizar más casos, producir un conocimiento de apariencia más exhaustivo en el sentido de acumular información.

Como ha identificado Osborne (2008), para el caso español, la producción de las cifras ha sido un lugar de tensión central entre las organizaciones feministas y las entidades estatales. Osborne analizando "los números de las violencias" identifica que las organizaciones consiguieron exhibir año a año números más abultados que las dependencias estatales a partir de una ampliación de las categorías de víctima, victimario y de la conducta considerada violenta. Esa amplia-

militancias feministas latinoamericanas a partir de la década de los '90, señalando el proceso de institucionalización del feminismo. (Sonia Álvarez et al. 2003) 
ción es entendida, no como una modificación de los criterios de contabilidad que resultan de una reelaboración de los marcos de interpretación, sino como efecto de un estudio más acabado, más completo, más verdadero. En el caso local esta operación se produce de modo similar para los casos de feminicidio. De hecho, en el desarrollo del informe van proliferando los criterios de inclusión para que la categoría femicidio pueda contener más casos mientras que se encuentran ausentes criterios de exclusión que puedan acotar los límites del fenómeno definido. Por ejemplo, mientras que La Casa del Encuentro dispone únicamente de la categoría femicidio para interpretar las muertes violentas de mujeres, en otros registros se definen y deslindan distintos tipos de muertes y se diferencia la categoría de "mujeres asesinadas" de "femicidios".

Con un efecto similar, el informe recupera la categoría clásica de Diana Russell de "femicidio vinculado" - la cual originalmente tuvo el espíritu de expandir la categoría para incorporar a las personas asesinadas por quedar expuestas en la "línea de fuego" del agresor - para nuevamente ampliarla incluyendo aquellos casos que pudieran ser considerados "represalias" indirectas a las mujeres (por ejemplo, episodios de asesinatos a hijos/as, familiares o afectos de aquellas). Así, en el informe quedan subsumidos a los marcos interpretativos de la figura expansiva de femicidio los asesinatos de menores por parte de adultos (padres, padrastros, etc) que antaño eran analizados como infanticidios. Lo que en tiempos anteriores se explicaba bajo el paradigma de la violencia doméstica o maltrato infantil ahora experimenta un desplazamiento interpretativo hacia el paradigma de la violencia de género.

Como es bien sabido las viejas conceptualizaciones, ligadas a la llamada violencia doméstica, habían demostrado su insuficiencia e, incluso, su fraude en numerosas ocasiones, en tanto y en cuanto, actuaban como eufemismos estratégicos que invisibilizaban las violencias específicas padecidas por las mujeres (Daich, 2012). Ahora bien, esta nueva perspectiva que representa una avance en tanto que consigue visibilizar la violencia producida contra las mujeres como una violencia específica que amerita ser pensada y denunciada, por otro lado, tiende a reconfigurar la lectura de la conflictividad social e interpersonal en torno al vector de opresión género como condición a priori determinante por sobre otras, reduciendo la interseccionalidad operante en toda situación (García Selgas y García García, 2008). De esta manera, el empleo de figuras como "femicidio vinculado" termina por reducir las complejidades de cada situación, al subordinarse a la lógica de la producción de cifras que demanda únicamente un crecimiento de los casos y no una mayor agudeza analítica.

El segundo procedimiento implicado en los observatorios se vincula a la recolección de casos con miras a la cuantificación del fenómeno, lo cual acarrea la producción de una veracidad sustentada más en la magnitud numérica de la información que en la precisión, refinamiento y articulación de las categorías. En

9 Tal como los informes confeccionados por Feminicidios.net (España) a partir de 2011 Disponibles en https://feminicidio.net/ (consultado el 3/12/2018) 
la lógica de los observatorios, cuando hablamos de cifras, cuanto más grande, siempre mejor es. La grandilocuencia del número actúa intensificando su verdad.

Este procedimiento constituye el núcleo central del informe. Los gráficos de barras y circulares en los que se exponen los resultados, dominan por sobre el relato de los casos, apenas descriptos, que son compilados al final del informe en un anexo. La estética de los números, los porcentajes y los gráficos son centrales para que las cifras aparezcan como un artefacto que puede, naturalmente, reducir las experiencias a eventos discretos, estandarizables y, por ende, cuantificables. Este proceder forma parte de lo que Engle Merry (2016) denomina "cultura del indicador" la cual otorga gran valor a los datos numéricos como una forma para conocer la realidad y producir intervenciones sobre ella.
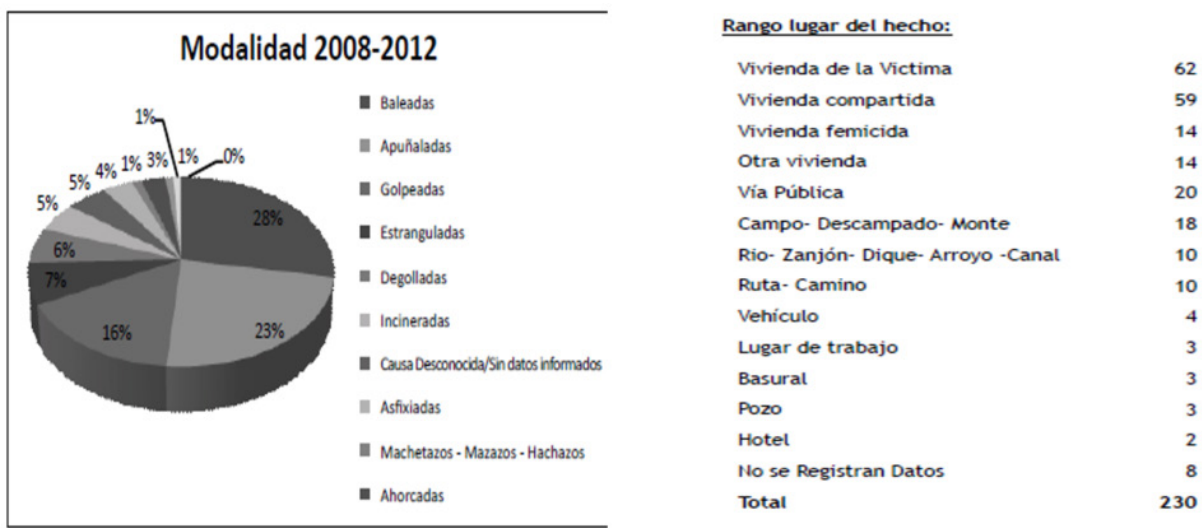

Fuente: Casa del Encuentro, "Por ellas. 5 años de Informes de femicidios", pág. 71 y 72

El informe presenta desagregados los datos por modalidad y por lugar del hecho que multiplican los valores que puede asumir un indicador. Por ejemplo, en el caso de modalidad, se contempla: "baleadas", "apuñaladas", "golpeadas", "estranguladas", "incineradas", "[por] causa desconocida/sin datos informados", "[por] Machetazos-mazazos-hachazos", entre otras. La prolífera clasificación de las modalidades, sin embargo, no se somete a análisis alguno a lo largo del informe, a excepción del que refiere a las asesinadas a efecto del uso del fuego que tiene dedicado un capítulo entero sobre el que volveremos más adelante.

Por otra parte, los valores del indicador de "lugar del hecho" son también profusos y si bien contemplan "vivienda de la víctima", "vivienda compartida" y "vivienda femicida", también permiten la siguiente (in)clasificación: "campodescampado-monte", "río-zanjón- dique-arroyo-canal", "ruta-camino", "basural", "pozo", "hotel". La lógica de la construcción de las categorías multiplica los detalles en torno a la escena del crimen y contribuye a intensificar el relato. Como en el caso anterior, esta anatomopolítica del detalle no encuentra un análisis que pueda organizarla y jerarquizarla. De hecho, mientras algunos valores están desprovistos de representatividad estadística, otros se yuxtaponen entre sí. 
Por otra parte y paradójicamente, los datos construidos no contemplan otras variables como ser: desde la condición socioeconómica hasta, por ejemplo, el considerable porcentaje de suicidios de los femicidas post factum. ${ }^{10}$ Este último nos refiere a los numerosos casos, consignados en el anexo del informe, en los que el femicida termina por acabar con su propia vida en el marco del mismo hecho ${ }^{11}$. De este modo, a excepción de la condición de género (considerada a priori determinante) y el vínculo con el agresor, se borran del análisis distintos patrones de riesgo y las lógicas que pudieran estar implicadas, y toman su lugar elementos propios de una escópica criminalística atenta a la escena y mecánica del crimen.

Para Dávila (1996) la cuantificación no es una cuestión de números sino de orden. El orden es la interpretación (negada) que organiza conceptualmente la perspectiva, a partir del cual se incluyen algunas dimensiones y se excluyen otras en miras a una estandarización del mundo social. Ellosupone una interpretación de los acontecimientos que pone en relieve ciertos aspectos de los mismos, al tiempo que deja caer en el olvido otros. En este caso, la estandarización supone la producción de "hechos" que se recortan de una trama social de relaciones, la delimitación de "eventos" que se abstraen de una historia de densa urdimbre y la descripción puntillosa de "escenas del crimen" que suspende los contextos sociales y económicos.

Finalmente, el tercer procedimiento es el de la visibilización. En su acepción nativa, la visibilización es una meta buscada como un modo de develar una realidad negada o ignorada que antecede a la mirada. En nuestro análisis la visibilización es entendida como un proceso de construcción/invención de nuevos objetos que permite producir una atención pública sobre ellos, interpelando a los sujetos e incitándolos a actuar.

La visibilidad está ligada a la potencialidad que detentan los números para capilarizarse a efectos de la estética que los presenta, la ilusión de su transparencia y la idea de su correspondencia con el mundo social. Las generalizaciones que se desprenden del método inductivo así aplicado se cristalizan en leyes con pretensiones predictivas que permiten sintagmas del estilo "una mujer es asesinada cada 30 horas" (luego "cada 21 horas" y "cada 18 horas"). En este procedimiento se anudan el gesto político de la denuncia y la resolución del método científico.

${ }^{10}$ Osborne (2009) advierte sobre la necesidad de combatir el mito de que solo las mujeres pobres sufren violencia de género, pero al mismo tiempo reclama la consideración de distintos factores de riesgo o de vulnerabilidad que afectan diferencialmente a las mujeres.

${ }^{11}$ El porcentaje importante que representan los suicidios de los femicidas presentados en el anexo del informe, habilita otro arco de preguntas en torno a cuáles serían las herramientas de intervención pertinentes. García Selgas y García García (2008), analizando el caso español, llaman la atención sobre la subestimación que los estudios e informes sobre violencia de género expresan en torno a las transformaciones de las relaciones sexo afectivas de las últimas décadas, y la consecuente inestabilidad e incertidumbre que significado para las relaciones heterosexuales. Para estos autores, esta subestimación compromete el diseño de dispositivos de intervención. 
Más allá de cualquier patrón de riesgo, estos sintagmas promueven identificaciones en el marco de un discurso de alarma social, que convierte a todas las mujeres (heterosexuales) en potenciales víctimas. Son narrativas que no sólo exhiben un problema sino que interpelan a los sujetos modelando la interpretación de sus experiencias pasadas, presentes y futuras. Cabría preguntarse si no estamos frente a un tipo de discurso que actúa al modo de una tecnología de género, abonando a la reproducción de una matriz heteronormativa, es decir, que demanda una determinada actuación y destino para sus partes, más que una herramienta de transformación de lo existente.

\subsection{El orden de la interpretación}

Como señalamos más arriba las cifras detentan un carácter persuasivo a partir de la aparente escisión entre interpretación y descripción. Esta potencia de los números los ha constituido como elementos privilegiados para la construcción de un "hecho social" (Poovey, 1998), dispuestos a ser rápidamente instrumentalizados en los dispositivos de gubernamentalidad. Ahora bien, detrás de las cuentas se esconde un orden de interpretación que estructura el significado de los números que es pocas veces sometido a escrutinio.

Así en su caracterización de la violencia sexista, el informe presenta el siguiente encadenamiento de hechos:

"Cuando comienza la relación de pareja, el proceso de enamoramiento, con sus idealizaciones de amor romántico, esconden a menudo el potencial violento o dominador del varón. De hecho, éste no se hace evidente hasta que la relación de pareja se ha "consolidado" de forma más estable, momento en el que el maltratador percibe la existencia de mayores lazos que atan a la mujer a la relación (...) y que dificultarán su marcha, sintiéndose con más poder y derecho a dominar y someterla. La aparición de formas tempranas de agresión verbal o psicológica (...) pueden ser el precedente de la posterior aparición de las primeras agresiones físicas o la agravación de las agresiones psicológicas. (...) Estas primeras agresiones no son visibilizadas (...), siendo naturalizadas en un contexto cultural patriarcal y machista, hasta que alcanzan sus niveles más severos y evidentes, con agresiones psicológicas graves y manifestaciones físicas de la violencia. Cuando estos maltratos severos son visibilizados, la dependencia y la sumisión de la mujer es tal que es mucho mayor la dificultad para salir de la relación violenta." (2013: 22)

Esta analítica se declara deudora de la teoría de Leonor Walker sobre los "ciclos de la violencia" en las parejas heterosexuales. En estas líneas se evidencia el desplazamiento de viejas claves interpretativas como "el crimen pasional" -en su formulación mediática- o la "emoción violenta" -en su formulación psicojudicial- y se dibuja una nueva figura: el varón violento. En este movimiento el violento emerge como un sujeto de una racionalidad extremadamente instrumental. Los modos y momentos bajo los cuales esta violencia se despliega parecen estar dominados por una cuidadosa estrategia que se despliega en etapas. 
Grafica claramente la trama de esta interpretación el lugar central que el informe les adjudica a los asesinatos con uso de fuego para producir una explicación sobre la violencia sexista. Los femicidios por uso de fuego son un tipo infrecuente, es decir, que no podrían tomarse como representativos. Podríamos especular que la centralidad de este tipo de femicidios en el informe está asociada a la espectacularización que el mismo supone y al impacto que, por consecuencia, estos casos han tenido en los medios de comunicación masivos. Pero, además, las autoras declaran que estos femicidios son seleccionados porque representan el "caso paradigmático" de la violencia extrema hacia las mujeres. En tanto y en cuanto no representa la mayoría de los casos, la condición paradigmática de estos femicidios estaría dada por la posibilidad que éstos tienen para revelar las lógicas e intenciones de sus perpetradores.

En el informe argumentan que quien comete este tipo de delitos aspira a conseguir impunidad desplegando una racionalidad instrumental para tal fin. Bajo esta interpretación: "[e]l fuego destruye, haciendo desaparecer pruebas indicadoras de un ataque, y en el imaginario de estos varones violentos se instala la sensación de lograr "el crimen perfecto"” (2013: 53). Entonces, y a contrasentido de cualquier análisis pericial, este tipo de femicidios son considerados como aquellos que permiten borrar todas las pruebas del hecho y garantizar la impunidad del asesino. Casado Aparicio (2012) analizando las interpretaciones en torno a la figura del "maltratador" en los discursos críticos sobre la violencia de género en el caso de España, señala que las mismas se sostienen en una asociación directa no problematizada entre cálculo racional, control, persuasión, estrategia, intencionalidad y masculinidad. Esta asociación puede verse también en el caso analizado.

Casado Aparicio (2012) argumenta que dichas interpretaciones funcionan "haciendo el género" más que dando cuenta de la complejidad de los acontecimientos que rodean a la violencia de género. La autora señala la simplificación que produce en el análisis de la violencia la figura y las ficciones en torno al "maltratador". Como sostiene esta autora para el caso de los "maltratadores", la rigidez de la matriz interpretativa sobre los casos de femicidio podrían también comprometer los diagnósticos del problema y la eficacia de las intervenciones destinadas a combatirlo.

\section{A MODO DE CONSIDERACIONES FINALES}

En este trabajo nos hemos propuesto explorar los supuestos epistémicometodológicos de los procesos de producción de cifras de femicidios llevados adelante por la ONG la Casa del Encuentro, pionera local en esta tarea. Nos ha interesado señalar los procedimientos performáticos de objetividad a partir de las cuales estos discursos se construyen: el despliegue de un método positivista inductivo y una reificación de los resultados a partir del empleo de las cifras como evidencia indiscutible de los "hechos". 
Los diseños cuantitativos participan con facilidad de las ficciones de objetividad al tiempo que son rígidos y cerrados; no permiten el ingreso de información no prevista (Dávila 1996, Besse 2011). En este sentido, y desde una perspectiva histórica, sorprende la alianza entablada entre algunos feminismos y lo que Dávila (1996) denomina el imperialismo cuantitativista, puesto que los discursos científicos han sabido construir su valía a partir de la exclusión sistemática de las experiencias incuantificables y depreciadas de las mujeres y otras poblaciones abyectas. Pero, entonces, ¿cómo hacer lugar a la ambivalencia y complejidad de la experiencia? ¿Cómo construir una disposición abierta a lo no conjeturado?

Desde las epistemologías feministas, que trabajosamente aprendimos a construir, entendimos el valor de reclamar un método de conciencia epistémica sobre las ficciones y las condiciones sobre las que el conocimiento se produce. Reconocimos la importancia de apostar a un método que rehuyera a la tentación de una mirada des-encarnada, truco de los dioses, en pos de la producción de un conocimiento situado, que reconociera tanto la parcialidad de su perspectiva como la condición política de su enunciación (Haraway, 1995; Pedrero Nieto, 2010).

En la contemporaneidad, la glotonería de la vista (Haraway, 1995) y el poder mágico que para nuestras sociedades tienen las cifras (Best, 2001) han estimulado la producción de "observatorios de la violencia" como un modo de validar la denuncia histórica del feminismo. La política de cifras se ha convertido en un modo privilegiado de disputar legitimidad y recursos tanto en el campo social y de organizaciones sociales como en las burocracias estatales. Así, la política de cifras se muestra como una vía para la constitución de experticias. De hecho, la multiplicación de observatorios ha creado la condición de posibilidad para la experticia feminista y, por tanto, una jerarquización de saberes. Parafraseando a Spivak podríamos preguntarnos ¿puede hablar quien no tiene una cifra?

Finalmente, la incorporación de la "política de cifras" -y su experticia- a la lengua de denuncia del feminismo puede ser interpretada, en algún sentido, como un triunfo del movimiento, pero vale preguntarnos por lo que se pierde en esta operación. ¿Las feministas no deberíamos mantener ciertas alertas ante los riesgos de las clausuras analíticas propias de estos modos de producción de conocimiento? No se trata de rechazar cualquier estrategia de investigación que se sirva de la cuantificación como herramienta pero sí de advertir sobre la subordinación a una lógica de mera captura y suma de casos.

La visibilización del relato cifrado de los femicidios ha conseguido instalar una demanda en la agenda pública e, incluso, estatal, pero éste en tanto y en cuanto oblitera todo tipo de información contextual y singular de cada suceso tiene el efecto de producir una interpretación que pierde de vista la importancia de una analítica interseccional. Asimismo, la estrategia de visibilización de las violencias en éstos términos actúa paradójicamente como tecnología de género que refuerza guiones heteronormados al asociar indiscutidamente los varones a la racionalidad cruel, a la voluntad de control y a la agencia libre. Estas narrativas no sólo producen scripts de géneros, sino que comprometen los diagnósticos sobre la violencia de género y las intervenciones para tratarla. 
Por otro lado, la subordinación a la cuantificación que reduce la trama de la violencia a eventos discretos organizados en torno a la díada -simplista, estática y a priori- de víctima y victimario, tiene el efecto de limitar nuestra imaginación política feminista a intervenciones centradas en los poderes punitivos del estado. Como sostiene Tamar Pitch (2003 [1989]) muchas veces no se trata tanto de discutir los términos de la solución como de repensar las matrices de inteligibilidad que definen el problema. En el caso que hemos analizado, si bien la lógica de la política de cifras consigue revelar matrices de opresión hacia las mujeres, parece proyectarse hacia una solución punitiva. Es por esta razón que resulta fundamental abonan a una reflexión epistemológica que esté dispuesta a conmover la mirada.

\section{BIBLIOGRAFÍA}

ÁLVAREZ, S.; FRIEDMAN, E.; BECKAMN, E.; BLACKWELL, M.; STOLTZ CHINCHILLA, N.; LEBON, N.; NAVARRO, M.; RÍOS TOBAR, M. (2003): "Encontrando os feminismos latino-americanos e caribenhos", Revista Estudos Feministas, 11 (2), 541-575.

ANDREAS, P. Y GREENHILL, K. M. (2010): "Introduction. The Politics of Numbers", Sex, Drugs, and Body Counts: The politics of number in global crime and conflict, Ithaca, Cornell University Press, pp. 1-22.

ARADAU, C. (2013): "Human trafficking between data and knowledge". Conferencia Data protection and right to privacy for marginalized groups: a new challenge in anti-trafficking policies, 25-27 Septiembre, Berlin, s/p.

BESSE, J. (2011): "Proceso y diseño en la construcción del objeto de investigación: las costuras de Frankenstein o un entre-dos que no hace dos", Epistemología fronteriza. Puntuaciones sobre teoría, método y técnica en ciencias sociales. Buenos Aires, Eudeba, pp. 93-114.

BEST, J. (2001): Damned Lies and Statistics. Untangling Numbers from the Media, Politicians, and Activists, Berkeley, University of California Press.

CARCEDO, A.; SAGOT, M. (2002): Femicidio en Costa Rica 1990-1999, San José, Instituto Nacional de las Mujeres.

CASA DEL ENCUENTRO (2013): Por Ellas... 5 años de Informes de Femicidios Observatorio de Femicidios en Argentina "Adriana Marisel Zambrano", Asociación Civil La Casa del Encuentro, Buenos Aires.

CASADO APARICIO, E. (2012): "Tramas de la violencia de género: sustantivación, metonimias, sinécdoques y preposiciones", Papeles del CEIC, vol. 2012/2, no 85, CEIC (Centro de Estudios sobre la Identidad Colectiva), Universidad del País Vasco, disponible en: http://www.identidadcolectiva.es/pdf/85.pdf, [consulta 9-112020]

DAICH, D. (2012): "Género y violencia: el honor en la tramitación judicial de las lesiones", Dilemas: Revista de Estudios de Conflicto e Controle Social, vol 6, n 1, pp. 103-126.

DÁVILA, A. (1996): "Las perspectivas metodológicas cualitativas y cuantitativas en las ciencias sociales: debate teórico e implicaciones praxiológicas", El análisis de la realidad social. Métodos y técnicas de investigación, Madrid, Alianza, pp. 69-83. 
DE MIGUEL ÁLVAREZ, A (2005): "La construcción de un marco feminista de interpretación: la violencia de género", Cuadernos de Trabajo Social, 18, pp.231-248, disponible en: <http://revistas.ucm.es/index.php/CUTS/article/view/8440>, [consulta: 27-12-2018]

ELÍAS, N. (2009): El proceso de la civilización, Barcelona, Fondo de Cultura Económica.

ENGLE MERRY, S. (2016): The seduction of quantification. Measuring human rights, gender violence and sex trafficking, Chicago, The University of Chicago Press.

FOUCAULT, M. (2006[1978]): Seguridad, Territorio, Población, Curso en el College de France (1977-1978), Buenos Aires, Fondo de Cultura Económica.

FOUCAULT, M. (2002 [1976]). Historia de la sexualidad. Voluntad de saber, Buenos Aires, Siglo XXI.

FOUCAULT, M. (1999). El orden del discurso, Madrid, Tusquets

GARCÍA FERRANDO, M. (1996): "La encuesta cuantitativa", El análisis de la realidad social. Métodos y técnicas de investigación, Madrid, Alianza, pp. 147-178.

GARRIGA ZUCAL, J. Y NOEL, G. (2010): “Notas para una definición antropológica de la violencia: un debate en curso", Publicar. En antropología y ciencias sociales, 9, pp. 97-121.

GATTI, G. (2017): Un mundo de víctimas, Barcelona, Antrophos.

GARCÍA SELGAS, F y GARCÍA GARCÍA, A. (2008): "La violencia de género y la investigación social", Violencia de género en las parejas heterosexuales: análisis, diagnóstico y problemas de intervención, Madrid, Consejería de Empleo y Mujer, pp.37-66.

GREGORI, M. F. (2010): "Violence and gender: Political paradoxes, conceptual shifts", Vibrant. Virtual Brazilian Anthropology, 7(2), pp. 216-235.

HALLEY, J., KOTISWARAN, P., THOMAS, C., SHAMIR, H. (2006): "From the International to the Local in Feminist Legal Responses to Rape, Prostitution/Sex Work, and Sex Trafficking: Four Studies in Contemporary Governance Feminism", Harvard Journal of Law and Gender 29 (2), pp. 335-423.

HARAWAY, D. (1995) Ciencia, cyborgs y mujeres. La reinvención de la naturaleza, Madrid, Cátedra.

LAGARDE, M. (2008): “Antropología, feminismo y política: violencia feminicida y derechos humanos de las mujeres" Retos teóricos y nuevas prácticas, San Sebastián, AnkulegiAntropologiaElkartea, pp. 211-229.

MONÁRREZ FRAGOSO, J. E. (2009): Trama de una injusticia. Feminicidio sexual sistémico en Ciudad Juárez, México, El Colegio de la Frontera Norte/Porrúa.

OSBORNE, R. (2008) Las cifras de la violencia Empiria. Revista de Metodología de Ciencias Sociales. N.o 15, enero-junio, 2008, pp. 99-124.

OSBORNE, R. (2009) Apuntes sobre violencia de género, Edicions Bellaterra, Barcelona.

PEDRERO NIETO, M. (2012): "Metodología cuantitativa para reforzar estudios cualitativos", Investigación feminista. Epistemología, metodología y representaciones sociales, México, Clacso, pp. 239-270.

PITCH, T. (2003 [1989]). Responsabilidades limitadas. Actores, conflictos y justicia penal. Buenos Aires, Ad-Hoc.

POOVEY, M. (1998): "A history of the modern fact: problems of knowledge in the sciences of wealth and society", Chicago, The University of Chicago Press.

RIFIOTIS, T. (2015 [1997]): "En los campos de la violencia: diferencia y positividad", AVÁ, 27, pp.103-116. 
RUSSELL, D. y Radford, J. (eds.) (2006): Feminicidio. La política del asesinato de las mujeres, México, Universidad Nacional Autónoma de México.

SEGATO, R. (2006): "Qué es un feminicidio. Notas para un debate emergente", Serie Antropología, 401.

TONKONOFF, S. (2014): "Prólogo", en Violencia y cultura: reflexiones contemporáneas sobre Argentina, Buenos Aires, Clacso, pp. 15-30.

TREBISACCE, C. (2020): "Un nacimiento situado para la violencia de género. Indagaciones sobre la militancia feminista porteña de los años 80", Anacronismo e Irrupción, Vol. 10, N¹8, 2020, pp. 118-138.

TUANA, N. (2006): “The speculum of ignorance: the women's health movement and epistemologies of ignorance", Hypatia, 21(3), pp. 1-19. 
